\title{
Sur quelques Collemboles cavernicoles de Roumanie
}

\author{
Par Magdalena Gruia ${ }^{1}$ )
}

Avec planches 19 (1) - $22(4)$

En étudiant le matériel de Collemboles cavernicoles recueilli dans les régions d'Olténie et de Hunedoara, nous avons rencontré quelques espèces intéressantes sur lesquelles notre attention s'est arrêtée et qui forment le sujet de cette note. Nous y décrivons une espèce nouvelle de Onychiuridae-Onychiurus orghidani n.sp. ${ }^{2}$ ). Possédant un matériel extrêmement riche des Beckerella décrites par G. N. Ionesco: $B$.quadriocellata et B. spelaea ${ }^{3}$ ) nous avons essayé d'élucider le problème posé par ces deux espèces.

La note contient aussi la discussion d'une espèce nouvelle pour notre pays 0. boldorii ainsi que la dispersion dans les grottes de Roumanie d'une espèce très fréquente de Lepidocyrtus - L.serbicus.

\section{Mesachorutes ojcoviensis Stach}

Distribution géographique: Pologne, G.S.S.R., Roumanie, Autriche, Italie et Espagne.

Distribution en Roumanie:

Région de Banat: La grotte "Gaura Ungurului»: 1155 - 28. VI. 1962 ؛ 2999 - 16. V. 1962 ; la grotte «Gaura cu muscă»: 1095 - 10. VI. $1962 ; 1759$ 13. VI. 1965 ; la grotte "Gaura Ponicovei»: $1485-12$. IX. 1963. Leg. A. et Şt. Negrea.

\footnotetext{
1) Institutul de Speologie E. G. Racovitza, Str. Dr. Capsa 8, Bucuresti 35.

2) L'espèce d'Onychiurus a été dédiée à M. le Professeur Dr. T. Orghidan, Directeur de l'Institut de Spéologie «E. Racovitża».

${ }^{3}$ ) Je remercie M. Dr. H. Gisin pour ses précieux renseignements.
} 
Région d'Olténie : La grotte «Peştera Liliecilor de la Mînăstirea Bistrița» : 163 - 3. II. 1958 ; la grotte "Isverna»: 9. VII. 1963 ; la grotte «Fuşteica» : 1664 -10. XI. 1965 ; la grotte "Peştera Vacilor din Steiul Orzeştilor»: 404 - 1. VIII. $1960 ; 520$ - 14. I. 1961 ; la grotte «Peştera Mare de la Apa Moişti» : 1665 - 9. IX. $1965 ; 1985$ - 11. X. 1966 ; la grotte «Cloşani» : 1300 6. VI. $1963 ; 1303$ - 6. VI. 1963 ; la grotte "Topolnița» : 1081 - 8. VI. 1962 ; 1489 - 1. XI. 1963. Leg. A. Bălăcesco, V. Decou et Şt. Avram.

Région de Hunedoara: La grotte "Peştera lui Duţu»: 1994 - 29. X. 1966. Leg. Tr. Orghidan, M. Dumitresco, A. Bălăcesco.

Région de Crişana: La grotte «Peştera Meziad»: 1923 - 13. VIII. 1966. Leg. A. Bălăcesco.

Région de Dobrogea: La grotte «Peştera de la Stîncă» (Le mont Consul) : $1329-5$. X. 1963. Leg. M. Dumitresco.

C. N. Ionesco décrit en 1922 (9) dans les grottes «des districts de Vîlcea et de Mehedinți» Beckerella quadriocellata, espèce caractérisée par : la présence de deux ocelles de chaque côté de la tête ; l'organe postantennal de forme ovale ; l'absence d'appendice empodial et des épines anales. L'auteur ne parle point de la furca, fait qui a déterminé Stach à mentionner dans la diagnose de l'espèce l'absence de celle-ci.

Pendant 40 ans cette espèce a été placée dans divers genres : Hypogastrura (Mesogastrura) Gisin 1944, Subbeckerella Stach 1949 et Mesachorutes Gisin 1960 (la rendant synonyme de Mesachorutes ojcoviensis). In 1964, Palissa range de nouveau cette espèce dans le genre Mesogastrura.

Nous possédons un matériel formé de centaines d'individus des grottes : Fuşteica, Vacilor din Steiul Orzeştilor, Topolnița et Cloşani, des grottes du Plateau de Mehedinți, mais nous n'avons jamais trouvé Beckerella quadriocellata Ionesco, nos déterminations montrant la présence de Mesachorutes ojcoviensis. Tous les exemplaires possèdent l'organe postantennal formé d'une vésicule en forme de rosette, un appendice empodial et une furca identiques à ceux des individus décrits par Stach. Tout ceci confirme l'opinion du Dr. Gisin et du Dr. Palissa sur la synonymie des deux espèces : Beckerella quadriocellata et Mesachorutes ojcoviensis.

Nous signalons chez les exemplaires des grottes Isverna, Meziad et Gaura Ungurului l'absence de pigment dans les taches oculaires. On ne peut faire un rapprochement avec Mesachorutes tiliophilus Locksa (10) parce que la griffe est identique à celle de M.ojcoviensis et que la chétotaxie ne correspond pas, non plus.

En considérant les prises de guano faites par A. Bălăcesco et V. Decou (2) dans la grotte Vacilor din Steiul Orzeştilor, on a pu observer ce qui suit (les sondages ont été faits dans la zone centrale du tas de guano, sous la colonie de Rhinolophus blasii et Rh.euryale). 


\begin{tabular}{l|c|c|c}
\hline \multicolumn{1}{c|}{ Sondages } & $\begin{array}{c}\text { Profondeur } \\
\text { en cm. }\end{array}$ & $\begin{array}{r}\text { Volume } \\
\text { en } \mathrm{cm}^{\mathbf{3}}\end{array}$ & Nr. d'ex. \\
\hline Dans le guano frais & $0-3$ & 2.700 & 30 \\
10 jours après le & $0-3$ & 675 & 310 \\
départ de la colonie & $3-6$ & 675 & 200 \\
& $6-9$ & 675 & 30 \\
1 mois après le & $0-3$ & 1.225 & 460 \\
départ de la colonie & $3-6$ & 675 & 200 \\
& $6-9$ & 90 & 10 \\
& $9-12$ & 1.350 & 2
\end{tabular}

On remarque que $M$.ojcoviensis préfère les premières couches de guano aérées et plus sèches.

Echantillons de guano de la grotte Fuşteica sous la colonie Myotis myotis et M.capaccini.

\begin{tabular}{l|c|c|c}
\hline \multicolumn{1}{c|}{ Sondages } & $\begin{array}{c}\text { Profondeur } \\
\text { en cm. }\end{array}$ & $\begin{array}{c}\text { Volume } \\
\text { en cm. }\end{array}$ & Nr. d'ex. \\
\hline $\begin{array}{l}\text { Guano frais et humide } \\
\begin{array}{c}\text { Guano à surface couverte } \\
\text { de moisissures, d'une petite } \\
\text { plateforme alluvionnaire }\end{array}\end{array}$ & $0-6$ & 2.400 & 0 \\
$\begin{array}{c}\text { Guano non tassé, humecté } \\
\text { par la crue des eaux } \\
\text { souterraines }\end{array}$ & $0-3,5$ & 350 & 420 \\
$\begin{array}{c}\text { Guano sec, couvert de } \\
\text { moisissures }\end{array}$ & $0-2$ & 800 & 1100
\end{tabular}

On remarque que le plus grand nombre de Collemboles se rencontre dans les tas de guano ancien non humecté par l'eau et couvert de moisissures. Cela a été constaté aussi dans d'autres grottes (Duțu et Meziad), où, sur des petits monceaux de guano non tassé pullulent des Collemboles appartenant à l'espèce M.ojcoviensis. C'est donc bien une espèce guanobie.

\section{Xenylla spelaea (Ionesco)}

(Planche 1, 2)

Distribution géographique : Roumanie.

Région Olténie : La grotte "Isverna": 103 ex. - 8. VII. 1963. Leg. A. Bălăcesco et V. Decou. 
Région Crişana: La grotte «Meziad»: 60 ex. 1923 - 13. VIII, 1966 ; la grotte «Peştera Roşie din Valea Lazurilor»: 50 ex. 1929 - 11. VIII. 1966. Leg. A. Bălăcesco.

En 1922, C. N. Ionesco décrit l'espèce B. spelaea d'après deux exemplaires de la grotte Isverna qu'il attribue au genre Beckerella. Les individus ont été recueillis sur le guano, dans des endroits complètement obscurs et humides.

Comme B.quadriocellata, B.spelaea a été attribué ensuite à plusieurs genres : Acherontides Bonet 1946, Subbeckerella Stach 1949 (13) et Mesachorutes (Gisin 1960) (5). En 1964 Palissa suppose la synonymie de cette espèce avec Mesogastrura ojcoviensis (11) (syn. Mesachorutes ojcoviensis).

Nous possédons un matériel très riche de la grotte Isverna, obtenu par le triage au Tüllgren de quelques prises de guano.

En étudiant ce matériel nous avons remarqué la présence de deux espèces : l'une qui, à certains points de vue, correspond à la description donnée par Ionesco pour B.spelaea et l'autre à Mesachorutes ojcoviensis.

Les individus des deux espèces peuvent être facilement confondus, parce qu'ils sont complètement dépigmentés et qu'ils ont à peu près la même taille (environ $1 \mathrm{~mm}$.) et la même forme générale du corps. En observant les préparations microscopiques nous avons constaté qu'une partie des individus ont sur la dens 4 poils, d'autres 2 poils. Tous les exemplaires à 4 poils sur la dens présentent un organe postantennal (sous la forme d'une rosette à 4 vésicules) et 2 taches oculaires dépigmentées, peu visibles; le tibiotarse a 2 poils sensitifs en forme de massue et l'empodium est filiforme. Ce sont les caractères spécifiques de Mesachorutes ojcoviensis.

Les exemplaires à 2 poils sur la dens n'ont ni ocelles, ni organe postantennal et la griffe manque d'empodium ; donc, à l'exception de la furca, les autres caractères correspondent à ceux indiqués par Ionesco pour B.spelaea. Nous sommes d'avis que ces individus appartiennent au genre Xenylla, puisqu'ils ne présentent pas d'organe postantennal et d'appendice empodial et que la dens ne possède que 2 poils. Comme Ionescu a étudié seulement 2 individus et que les deux espèces, comme nous l'avons déjà dit, se ressemblent entre elles à première vue, nous pensons qu'il a observé la partie dorsale du corps de Xenylla spelaea et la face ventrale d'un autre exemplaire, qui appartenait cependant à l'espèce Mesachorutes ojcoviensis.

Description de l'espèce :

Longueur du corps : 0,6-0,9 mm. Couleur blanche. Tégument granulé. 
En étudiant un lot de 30 exemplaires de la grotte de Meziad, j'ai remarqué une grande variabilité de la chétotaxie de cette espèce ; de même, j'ai pu constater aussi les différences chétotaxiques qui existent entre les individus immatures et adultes.

Le second tergite thoracique présente la même disposition des poils chez les adultes et chez les immatures. C'est le seul caractère fixe, en ce qui concerne la chétotaxie de X.spelaea.

Nous remarquons :

- manque de microchète $m_{3}$, mais en échange présence de $m_{4}$,

- manque de microchète $\mathrm{la}_{1}$,

- manque de microchète $\mathrm{p}_{3}$,

$-\mathrm{S}_{\mathrm{s}}=\mathrm{P}_{4}$.

L'inéxistence de $p_{3}$ éloigne $X$.spelaea de toutes les espèces de Xenylla connues jusqu'à présent, la rapprochant des espèces du genre Frisea, Pseudachorutes et Anurida. Pourtant, il ne peut pas être question de la placer parmi ces genres, étant donné la présence de la plaque molaire dentelée caractéristique des Hypogastrurinae.

Individus adultes (pl. 1)

Longueur du corps chez les mâles : 0,6 mm., et pour les femelles : $0,7-0,9 \mathrm{~mm}$.

La chétotaxie de la partie dorsale de la tête est comparable à celle de X.brevisimilis var.mediterranea Gama. L'absence du $l_{2}$ et la présence des microchètes $l_{4}$ et $l_{5}$ la différencie de cette dernière.

$52 \%$ des individus ont cette disposition des microchètes.

$8 \%$ présentent aussi le $\mathrm{v}_{2}$, donc l'«area verticalis» comprend 2 rangées de microchètes $\mathrm{v}$ et $\mathrm{v}_{2}$. Le reste de $40 \%$ des exemplaires ont ces microchètes disposées asymétriquement. Parmi le matériel que j'ai étudié, seulement $15 \%$ des individus présente la microchète $\mathrm{a}_{0}$, pour le reste celle-ci manque.

Ier tergite thoracique : $40 \%$ présente $3+3$ microchètes et $60 \% 2+3$ microchètes.

II e ${ }^{\mathrm{C}}$ III tergite thoracique : La disposition des micro- et des macrochètes est la même sur les deux segments.

III e et IVe tergite abdominal : $\mathrm{S}_{\mathrm{S}}$ est toujours placée dans la position $\mathrm{P}_{4}$ et respectivement $\mathrm{P}_{6}$. J'ai remarqué de nombreuses asymétries ou la disparition totale des microchètes $p_{3}$ ou $p_{5}$. 
La plus évidente variation est présentée par le segment $\mathrm{V}$ abdominal. $60 \%$ des individus ont la microchète $\mathrm{p}_{2}$ placée entre $\mathrm{p}_{1}$ et $\mathrm{P}_{3}$, mais située à un niveau intermédiaire entre les rangées antérieures et postérieures des sètes. Pour $40 \%$ d'exemplaires $\mathrm{p}_{2}$ manque d'une manière asymétrique.

Le VIe segment abdominal présente seulement $\mathrm{a}_{\mathbf{1}}$. On n'a remarqué aucune corrélation entre les positions tellement variables des microchètes.

Cette variabilité est la seule observée chez cette espèce cavernicole.

\section{Individus immatures}

Longueur du corps : mâles : 0,5 mm., femelles : 0,6 mm.

Tous les individus présentent sur «area verticalis» une seule paire de microchètes $\mathrm{v}_{\mathbf{1}}$.

$70 \%$ exemplaires ont sur le tergite thoracique III $2+2$ microchètes et $30 \% 2+3$ microchètes.

Aucun individu ne présente $3+3$ microchètes. En tous les cas, $\mathrm{p}_{2}$ du tergite $\mathrm{V}$ abdominal manque.

En revenant aux autres caractères des adultes:

Le rapport des articles antennaires est : 7/7/10/15. Les organes sensitifs des $\mathrm{III}^{\mathrm{e}}$ et $\mathrm{IVe}$ articles antennaires sont représentés par la planche $2: 1,2$.

L'organe postantennaire et les ocelles manquent.

La griffe, allongée, forte, présente une dent située dans la moitié apicale du bord interne. A la base de la griffe, latéralement, il y a 2 poils, insérés à des niveaux différents. L'empodium manque. Le tibiotarse possède 2 poils sensitifs dorsaux.

Furca de 1,5 fois plus courte que la longueur de l'antenne. Le rapport entre le manubrium, la dens et le mucron $=7 / 13 / 5$. Le manubrium présente sur le côté ventral 6 poils et la dens 2 poils. Le mucron est pointu, avec une dent antéapicale; la lamelle ventrale est absente. Tenaculum à 3 dents (pl. $2: 4,5)$.

Les épines anales insérées sur 2 papilles granulées, mesurent approximativement $1 / 3$ du bord interne de la griffe (pl. 1).

Xenylla spelaea doit être classé dans le groupe des espèces chez lesquelles le mucron est séparé de la dens, mais même au sein de ce groupe l'absence d'ocelles et de macrochète $p_{3}$ sur le tergite thoracique II et la forme du mucron lui confèrent une position à part.

On a prélevé, dans la grotte Isverna, quelques prises quantitatives de guano, d'où il résulte que $X$. spelaea comme l'espèce précédente, préfère les premières couches de guano, plus sèches et plus aérées. 


\begin{tabular}{l|c|c|c}
\hline \multicolumn{1}{c|}{ Sondage } & $\begin{array}{c}\text { Profondeur } \\
\text { en } \mathrm{cm} .\end{array}$ & $\begin{array}{c}\text { Volume } \\
\text { en } \mathrm{cm}^{3} .\end{array}$ & Nr. d'ex. \\
\hline Dans la zone centrale de & $0-3$ & 1.200 & 16 \\
guano sous la colonie de & $3-6$ & 1.200 & 82 \\
Myotis myotis et & $6-9$ & 1.200 & 4 \\
M. capaccini & $9-12$ & 1.200 & 1 \\
& $12-15$ & 1.200 & 1
\end{tabular}

Tout comme dans la grotte d'Isverna, dans celle de Meziad, nous avons trouvé Xenylla spelaea en compagnie de M.ojcoviensis.

Si dans les grottes Isverna et Meziad le tas de guano est situé dans les profondeurs de la grotte et que par conséquent l'espèce vit dans la zone obscure et humide, dans la grotte «peştera Roşie din Valea Lazurilor», où fourmillaient des milliers d'exemplaires de Xenylla spelaea, le guano se trouve en pleine lumière, à la base de la paroi de l'entrée de la grotte. C'est une espèce typiquement guanobie.

\section{Onychiurus orghidani n.sp.}

(Planche 3)

Distribution en Roumanie : La grotte "Arnăuților» : 23 ex. 1998 20. VII. 1962 ; 2 ex. 2020 - 9. VII. 1966 ; région Olténie. Leg. D. Dancău, V. Decou, D. Danielopol.

Description de l'espèce :

Longueur du corps : 2,1-2,25 mm. Couleur blanche. Tégument finement granulé, spécialement à la base de l'antenne. La chétotaxie de la tête et du corps est très variable d'un individu à l'autre, on remarque seulement les poils du IIe tergite thoracique et du VIe abdominal qui ont la même position et la même forme (pour les 10 exemplaires étudiés pl. $3: 1,2$ ).

Pseudocelles dorsaux : 33/133/33333. La position des pseudocelles sur le bord postérieur de la tête, sur le Ier tergite thoracique, comme sur les tergites IV et $\mathrm{V}$ de l'abdomen est réprésentée sur la pl. 3: 1,2.

Pseudocelles ventraux : 1/000/1212. Le pseudocelle sur le Ier sternite abdominal est placé latéralement, à la base du tube ventral (pl. $3: 3$ ). Sur le II et IVe sternite les deux pseudocelles ont une position médiane et latérale et sur le IIIe sternite l'unique pseudocelle a une position latérale. Le sous-coxe possède 2 pseudocelles. 
L'organe sensitif du III e article antennaire est formé de 5 papilles coniques finement granulées, 2 sensilles en forme de grappe et 2 baguettes centrales (pl. $3: 4$ ).

L'organe postantennal présente 12-14 vésicules composées (pl. $3: 5$ ). Griffe allongée, avec ou sans dent interne. Lorsque celle-ci existe, elle est bien différenciée et placée au milieu du bord intérieur de la griffe. L'empodium filiforme, de la même longueur que la griffe (pl. $3: 6$ ).

Un rudiment de furca manque. Tube ventral avec 17 poils ordinaires. Les épines anales ne sont pas placées sur des papilles anales. Leur dimension est égale à la moitié de la longueur du bord interne de la griffe.

Il n'y a pas d'organe ventral mâle.

O.orghidani se rapproche de Onychiurus sensitious Handschin (8) espèce décrite en 1928 de la grotte Ledenika (Balcans) et O.normalis Gisin, décrite en 1949 en Suisse.

\begin{tabular}{|c|c|c|}
\hline O.sensitivus & O.orghidani & O.normalis \\
\hline $\mathrm{L} .=1,5 \mathrm{~mm}$ & $\mathrm{~L} .=2,1-2,25 \mathrm{~mm}$. & $\mathrm{L} .=1,16-1,9 \mathrm{~mm}$. \\
\hline $\begin{array}{l}\text { Ps. dorsaux: } 33 / 123 / \\
\quad 33333\end{array}$ & $\begin{array}{l}\text { Ps. dorsaux : } 33 / \mathbf{1 3 3 /} \\
\quad 33333\end{array}$ & $\begin{array}{l}\text { Ps. dorsaux : } 32 / 133 / \\
\quad 33343\end{array}$ \\
\hline Ps. ventraux:/111 & $\begin{array}{l}\text { Ps. ventraux: } 1 / 000 / \\
\quad 1212\end{array}$ & Ps. ventraux:2/-/0101 \\
\hline Soubcoxe : ?/1/1 & Soubcoxe : 2 & Soubcoxe : 1 \\
\hline $\begin{aligned} \mathrm{OA}= & 5 \text { papilles et } 2 \text { bul- } \\
& \text { bes sensitifs sous } \\
& \text { forme de grappe }\end{aligned}$ & $\begin{aligned} \mathrm{OA}= & 5 \text { papilles et } 2 \\
& \text { bulbes sensitifs } \\
& \text { sous forme de } \\
& \text { grappe }\end{aligned}$ & $\begin{aligned} \mathrm{OA}= & 5 \text { papilles et } 2 \\
& \text { bulbes sensitifs } \\
& \text { sous forme de } \\
& \text { grappe }\end{aligned}$ \\
\hline $\begin{aligned} \text { OPA }= & 10-12 \text { vés. } \\
& \text { composées }\end{aligned}$ & $\begin{aligned} \mathrm{OPA}= & 12-14 \text { vés. } \\
& \text { composées }\end{aligned}$ & $\begin{aligned} \mathrm{OPA}= & 20 \text { vés. com- } \\
& \text { posées }\end{aligned}$ \\
\hline Griffe/empodium $=3 / 4$ & Griffe/empodium $=1 / 1$ & Griffe/empodium $=1 / 2$ \\
\hline Griffe sans d. i. & $\begin{array}{l}\text { Griffe avec d. i. } \\
\text { Griffe sans d. i. }\end{array}$ & Griffe sans d.i. \\
\hline $\mathrm{AE} /$ Griffe $=3 / 4$ & $\mathrm{AE} /$ Griffe $=1 / 2$ & $\mathrm{AE}=$ Griffe \\
\hline O.V. absent & O.V. absent & O.V. absent \\
\hline
\end{tabular}

De ces 3 espèces, O. orghidani a la taille la plus grande. Au point de vue des pseudocelles dorsaux, l'espèce se rapproche de O.sensitisus, à l'exception du II e segment thoracique, sur lequel O.orghidani présente 3 pseudocelles au lieu de 2 . Les pseudocelles du côté ventral sont totalement différents. 
En ce.qui concerne le rapport entre AE et la griffe, il est plus petit par comparaison avec les deux autres espèces. L'empodium est égal à la longueur du bord interne de la griffe, à la différence les deux autres espèces chez lesquelles l'appendice empodial est plus petit. Il existe donc chez les 3 espèces un rapport inversement proportionnel entre les dimensions de l'empodium et les épines anales.

O.normalis est une espèce endogée, tandis que O.sensitiøus est considérée comme une espèce troglobie.

Les 25 exemplaires appartenant à l'espèce O.orghidani ont été recueillis dans la grotte Arnăuților, situées dans le massif Stogu-Vînturarița.L'espèce est cantonnée dans ce seul point. Dans la grotte Liliecilor de Mînăstirea Bistrița, grotte située dans le même massif, O.orghidani n'a pas été rencontrée. Nous ne saurions pas dire si c'est un troglobie ou non, parce que nous n'avons pas cherché à l'extérieur de la grotte si cette espèce pouvait vivre aussi dans le domaine endogé.

\section{Onychiurus boldorii Denis}

(Planche 4)

Distribution géographique: La grotte "Postoina».

Distribution en Roumanie:

Région de Hunedoara: La grotte «Cioclovina cu apă»: 4 ex. 1062 24. VIII. $1962 ; 6$ ex. 1311 -1. VIII. 1963. Leg. T. Orghidan, M. Dumitresco, St. Avram, M. Gruia.

Longueur du corps : 1,5-1,75 mm. En ce qui concerne l'organe antennal, postantennal (avec 9-14 vés. comp.) et l'épine anale, les individus recueillis par nous, sont identiques à ceux décrits par Denis.

Le nombre de pseudocelles, du côté dorsal ainsi que ceux du côté ventral, est cependant différent, à savoir :

Pseudocelles dorsaux : 32/033/3334-53 - Denis indique 4 pseudocelles sur le IVe tergite abdominal. Nous avons remarqué chez nos exemplaires certaines variations: 2 क présentent 4 pseudocelles sur le $\mathrm{IV}^{\mathrm{e}}$ tergite abdominal et 1 i a 4 pseudocelles sur le Ve tergite abdominal. Chez 1 ơ nous avons observé au cadre du même organisme sur le IVe tergite 4 ou 5 pseudocelles. Le reste des individus ont 5 pseudocelles.

Pseudocelles ventraux : 3/011/4222 - Les exemplaires décrits par Denis présentent $2 / 000 / 1212$ ou $3 / 000 / 2101$, on y rencontre donc certaines variations. Chez les individus recueillis par nous, nous n'avons 
pas remarqué de variations dans le nombre de pseudocelles du côté ventral.

La disposition des pseudocelles ventraux ressemble à celle de $O$. silvarius, à l'exception du premier sternite abdominal chez lequel la disposition des pseudocelles est identique à celle de O.closanicus.

Subcoxa avec 2 pseudocelles.

Griffe sans dent interne et sans dent latérale.

L'organe ventral mâle est formé de 4 poils sur le II ${ }^{\text {e }}$ sternite et 6 poils sur le IIIe sternite abdominal, dans la mème disposition que chez les exemplaires de Postoina (pl. 4:1).

Grâce à cette ressemblance dans la forme de l'organe ventral mâle, nous avons considéré nos exemplaires comme étant des 0 .boldorii, malgré la différence dans le nombre de pseudocelles.

Dans la grotte Cioclovina cu apă les individus ont été recueillis sur de grands blocs où l'eau ruisselait, à côté de Onychiurus n. sp. et de Lepidocyrtus serbicus. Espèce troglobie. Nouvelle pour la Roumanie.

\section{Lepidocyrtus serbicus Denis}

Distribution géographique: Jugoslavie, Roumanie.

Distribution en Roumanie:

Région de Banat: La grotte "Gaura Ungurului»: 1153 - 28. VI. 1962; grotte "Casa Lotrilor»: 1387 - 25. VI. 1963 ; la grotte «Peştera din dealul Colțan»: 1374-20. VI. 1963 ; la grotte "Abatajul lui Anton»: 1377 - 22. VI. 1963 ; la grotte "Gaura Pîrşului de la capu Baciului» : 1734-20. V. 1965 ; la. grotte «Peştera Cerbului» : 1789 - 29. IX. 1965 ; la grotte "Peştera Popovăț» : 174 - 3. X. 1961 ; la grotte «Peştera Comarnic» : 599 - 2. VII. $1961 ; 767$ 2. X. $1961 ; 1400$ - 24. III. $1963 ; 1721$ - 18. V. $1965 ; 1780$ - 28. IX. 1963 ; 1783 - 28. IX. 1963 ; la grotte "Peştera Racovitza» : 1763 - 26. IX. 1965 ; la grotte «Peştera Vraşca»: 1814 - 3. X. 1965 ; la grotte "Peştera Buhui»: 613 - 5. VII. $1961 ; 750$ - 28. IX. 1961 ; la grotte «Peştera Mărghitaş» : 861 14. IV. 1961 ; la grotte "Peştera Ponor-Plopa» : 625 - 7. VIII. $1961 ; 740-$ 26. IX. 1961 ; la grotte «Găurile lui Mihai II»: 1160 - 17. VIII. 1962 ; la grotte "Peştera mică de la Lacu Dracului»: 1552 - 12. X. 1964 ; la grotte «Peştera Iasînoca": 1113-13 - 13. VI. 1962 ; la grotte "Peştera de la Izvorul Mînzului» : 1085 - 9. VI. 1962 ; la grotte «Peştera de la Româneşti» : 1352 22. V. 1963 ; la grotte «Peştera din Cioaca Bîrtului» : 1370 - 25. V. 1963 ; la grotte «Peştera din Piatra Fetii» : 1373 - 24. III. 1963. Leg. L. Botoşăneanu, St. Negrea, A. Negrea.

Région d'Olténie : La grotte "Liliecilor de la Mînăstirea Bistrița": 8 - 10. X. $1963 ; 249$ - 22. XII. $1958 ; 523$ - 24. I. 1961 ; la grotte No. 3 de "Steiul Orzeştilor» : 436 - 20. X. 1960 ; le gouffre "Gaura Rîndunelei» : 1479 - 3. VII. 1961 ; le gouffre de «Foeroaga îngustă»: 651 - 30. VI. 1961 ; 
le gouffre de "Cracul cu Plopi»: 1256 - 11. X. $1962 ; 1489$ - 1. XI. 1963. Leg. V.Decou, A. Balacesco, St. Avram.

Région de Hunedoara: La grotte "Cioclovina cu apă»: $1062-24$. VIII. $1962 ; 1309$ - 29. VI. $1963 ; 1310$ - 30. VI. $1963 ; 1311$ - 1. VII. 1963 ; le gouffre de "Şesul Leordei»: 1460 - 2. XI. 1963 ; la grotte "Peştera de la Bordul Mare»: 1553 - 30. X. 1964; la grotte «Peştera de la Tecuri» : 976 22. V. $1962 ; 978$ - 24. V. $1962 ; 979$ - 24. V. 1962 ; la grotte «Gaura Oanei»: 1060 - 25. V. 1962 ; la grotte "Gura Cetății» : 259 - 10. IV. 1959 ; 980 25. V. 1962 ; la grotte «Cetatea Bolii»: 970 - 21. V. 1962 ; la grotte «Peştera de la Paroşeni" : 1312 - 4. VII. 1963 ; la grotte "Peştera lui Duțu» : 1281 15. III. 1963 ; la grotte "Peştera din Cheile Ampoiței» : 3 - 19. VI. 1964 ; la grotte No. 1 de «Iezerul Ighiel»: 273 - 29. V. 1959. Leg. M. Dumitresco, Tr. Orghidan, M. Georgesco, St. Avram, M. Gruia.

Région MureşAuton o mă Maghiară: Les grottes de Cheile Vîrghişului. La grotte No. 14 (Peştera mare de la Mereşti) : 78 - 23. I. $1951 ; 377$ - 3. IX. 1960 ; la grotte No. 8 (Peştera Calului) : 97 - 24. VI. 1957 ; la grotte No. 9 : 253 - 18. III. $1959 ; 898$ - 20. XI. 1961 ; la grotte No. 11 : 497 - 23. III. $1961 ; 1274-27$. IX. 1962 ; la grotte No. 18 : $106-16$. VIII. 1957 ; la grotte No. $20: 255-17$. III. 1959 ; la grotte No. $27: 112-25$. VIII. 1957 ; la grotte No. $35: 505-26$. III. 1961 ; la grotte No. $37: 157-22$. I. 1958 ; la grotte No. 41 : 901 - 19. XI. 1961 ; la grotte No. 42 : 902 - 19. XI. 1961 ; la grotte No. 45 : 199 - 11. VIII. 1958. Leg. M. Dumitresco, Tr. Orghidan, M. Georgesco.

L'espèce a été décrite par Denis dans les grottes de Serbie en 1933/36. Ultérieurement, en 1941, le mème auteur l'a synonymisé avec L. pallidus Rt. En 1965, sur un matériel provenant de Roumanie, Gisin (7) a reconnu $L$. serbicus comme une bonne espèce. (Le matériel provient de prises épigées.)

Lepidocyrtus serbicus est le Collembole le plus répandu dans les grottes des monts Carpates, prédominant dans les régions de Banat et Mureş Autonomă Maghiară, où il est l'espèce la plus fréquente. Il n'a pas été trouvé en Dobrogea. Troglophile.

\section{RÉSUMÉ}

L'étude d'un important matériel de Collemboles recueilli, en Roumanie, permet à l'auteur de présenter six espèces.

Une de ces espèces est nouvelle: Onychiurus orghidani n.sp. trouvée dans une grotte d'Olténie.

La présence en Roumanie d'Onychiurus boldorii Denis est signalée pour la première fois, et la découverte d'un grand nombre de Beckerella spelaea Ionesco permet de placer cette espèce dans le genre Xenylla.

Enfin, les répartitions géographiques de Mesachorutes ojcoviensis Stach et de Lepidocyrtus serbicus Denis sont complétées. 


\section{SUMMARY}

An important collection of Collembola from Romania contained six species.

One of them is new - Onychiurus orghidani n.sp. found in one cave of Oltenia.

The presence of Onychiurus boldorii Denis in Romania is reported for the first time, and the discovery of a large number of Beckerella spelaea Ionesco made it possible to place this species in the genus Xenylla.

Finally, additions are made to the known geographic distributions of Mesachorutes ojcoviensis Stach and Lepidocyrtus serbicus Denis.

\section{BIBLIOGRAPHIE}

1. Cassagnau, P. (1952) - Faune française des Collemboles. Quelques nouveaux Poduromorphes méridionaux. Bull. Soc. Hist. Nat. Toulouse, T. $87: 3-4$.

2. Decu, A., et Decu, V. (1964) - Sur la synusie du guano des grottes d’Olténie et du Banat. Annales de Spéléologie. T. 19, fasc. 4.

3. Denis, J. R. (1936) - Collemboles d'Italie (principalement cavernicoles). Boll. Soc. Adriat. Sci. natur. Trieste. T. 36 : 96-165.

4. Gama da, M. M. (1944) - Collembolas de Portugal Continental. Coimbra.

5. Gisin, H. (1960) - Collembolenfauna Europas. Genève.

6. - (1960) - Colembolles cavernicoles de la Suisse, du Jura français, de la Haute-Savoie et de la Bourgogne. Rev. Suisse de Zool. Tom. 67, fasc. I : $94-96$.

7. - (1965) - Nouvelles notes taxonomiques sur les Lepidocyrtus. Rev. Ecol. Biol. Sol. T. II, fasc. 4 : 519-524.

8. Handschin, E. (1928) - Mitteilungen aus den Königl. Naturwissenschaftlichen Instituten Sofias. Band I : 17-27.

9. Ionesco, C. N. (1922) - Quelques nouveaux insectes Collemboles récoltés dans les grottes des Carpates méridionales. Ann. Sci de Iassy. T. III, No. 4 .

10. Locksa, J. (1966) - Angaben zu den Kenntnissen über die Collembolenfauna des Bakory-Gebirges. Opusc. Zool. Budapest. T. VI, fasc. 1 : 139-156.

11. Palissa, A. (1964) - Die Tierwelt Mitteleuropas.

12. Rusek, J. (1965) - Beitrag zur Collembolenfauna Bulgariens. Acta Univers. Carolinae - Biologica, No. 2 : 179-191. 
13. Sтасн, J. (1949) - The Apterygotan fauna of Poland in relation to the world-fauna of this group of insects. fam. Neogastruridae.

EXPLICATIONS DES PLANCHES 19 (1)-22 (4)

\section{PLANCHE 19 (1)}

Xenylla spelaea (Ionesco). -1 . Chétotaxie dorsale du corp $(8 \times 40)$.

\section{PLANGHE $20(2)$}

1. Organe sensoriel du III e article antennaire $(15 \times 1000)$. -2 . Organe sensoriel du IVe article antennaire $(15 \times 40)$. -3 . Griffe de la IIIe patte $(15 \times 60)$. -4 . Furca - vue ventrale $(15 \times 40)$. -5 . Furca - vue latérale $(15 \times 60)$.

\section{PLANGHE 21 (3)}

Onychiurus orghidani n.sp. - 1. Chétotaxie et répartition des pseudocelles sur la portion postérieure de la tête et sur le premier tergite thoracique $(10 \times 20)$. -2 . Chétotaxie et répartition des pseudocelles sur les $\mathrm{IVe}^{\mathrm{e}} \mathrm{Ve}$ et VIe tergites thoraciques $(10 \times 20)$. -3 . Position du pseudocelle sur le Ier sternite abdominal $(15 \times 20)$. -4 . Organe sensoriel du III ${ }^{\mathrm{e}}$ article antennaire $(15 \times 40)$. -5 . Organe postantennaire $(15 \times 40) .-6$. Griffe de la III ${ }^{\mathrm{e}}$ patte $(15 \times 40)$.

\section{PLANCHE $22(4)$}

Onychiurus boldorii Denis. - 1. Organe ventral mâle $(15 \times 40)$. 

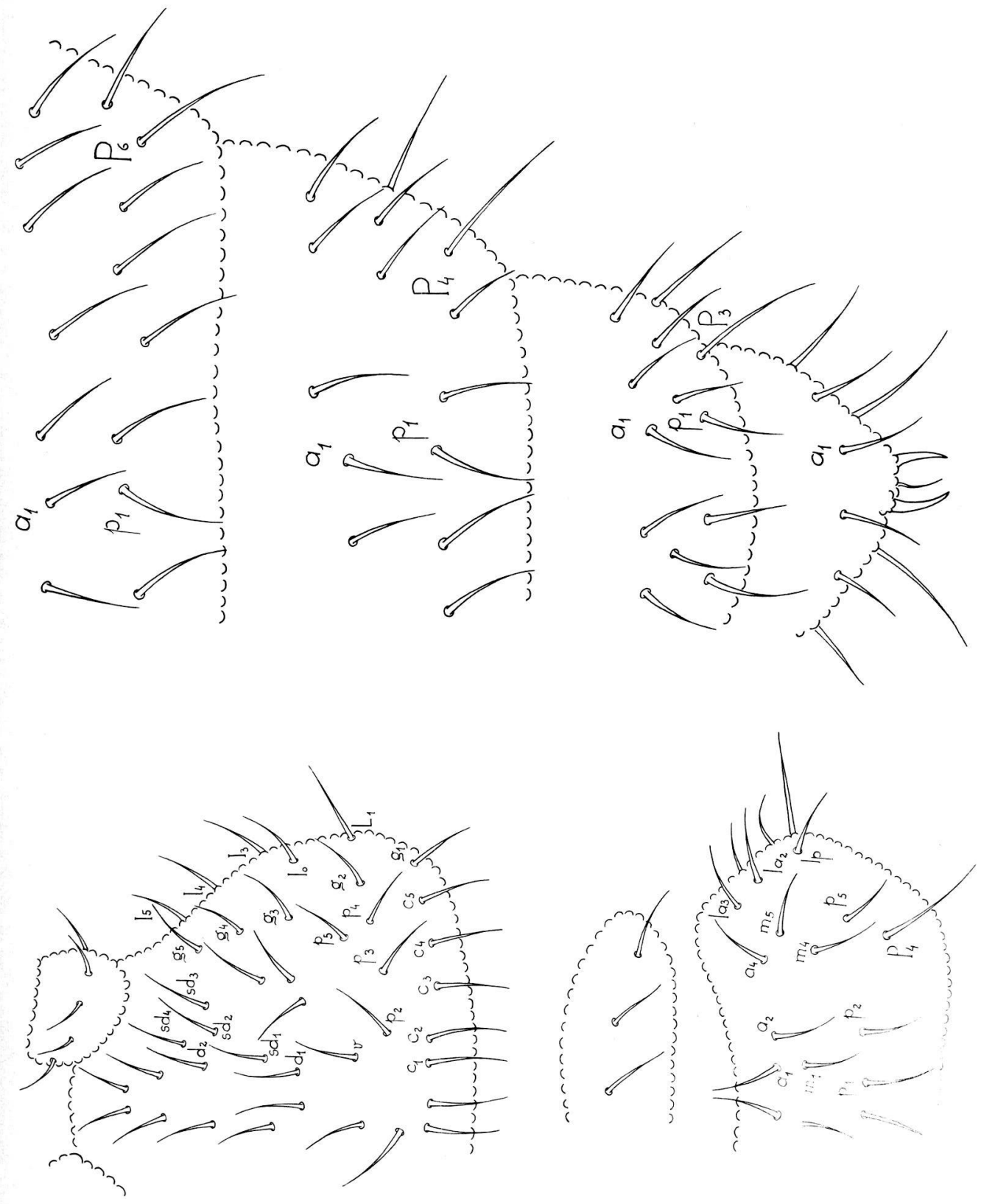

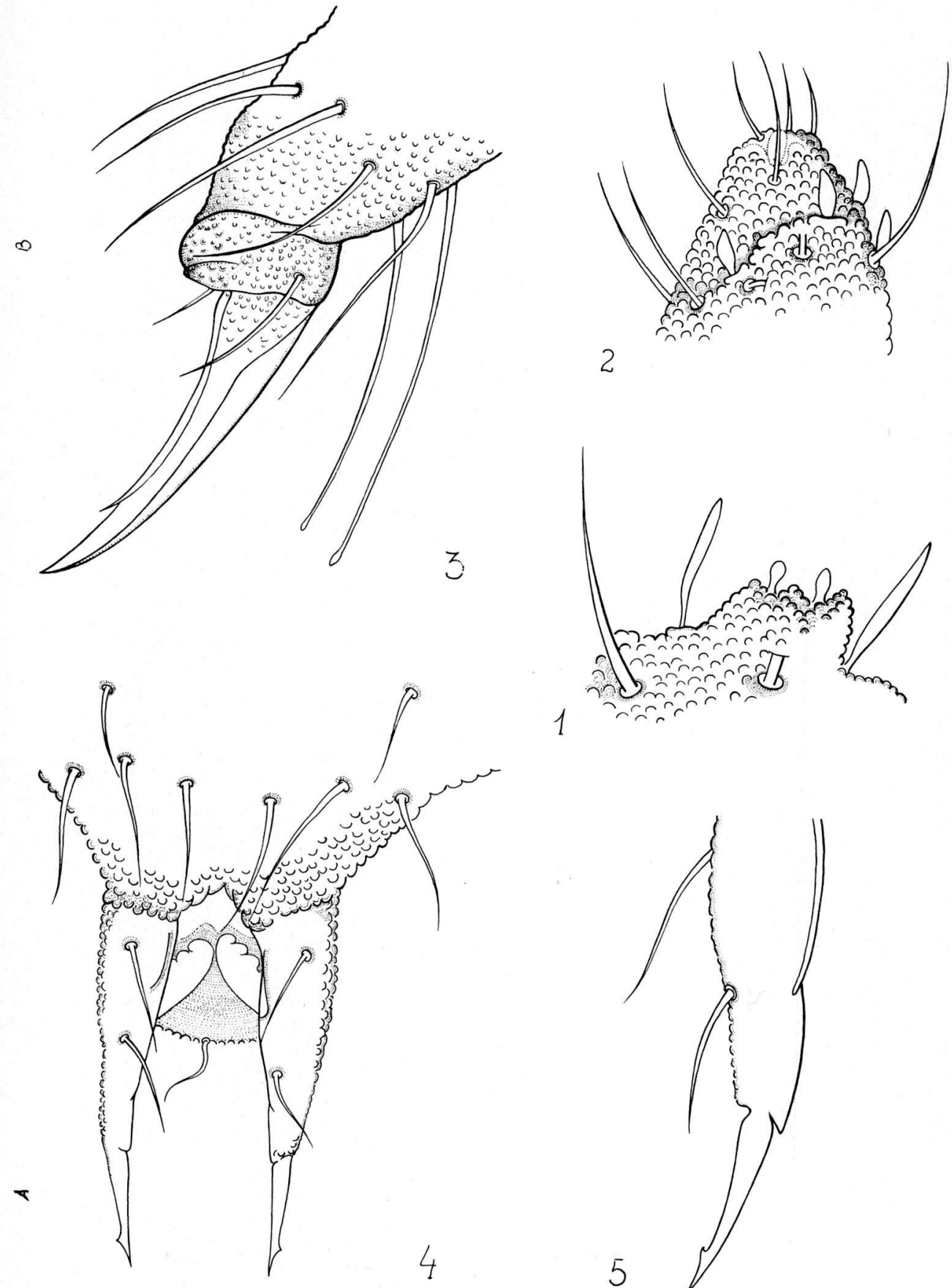

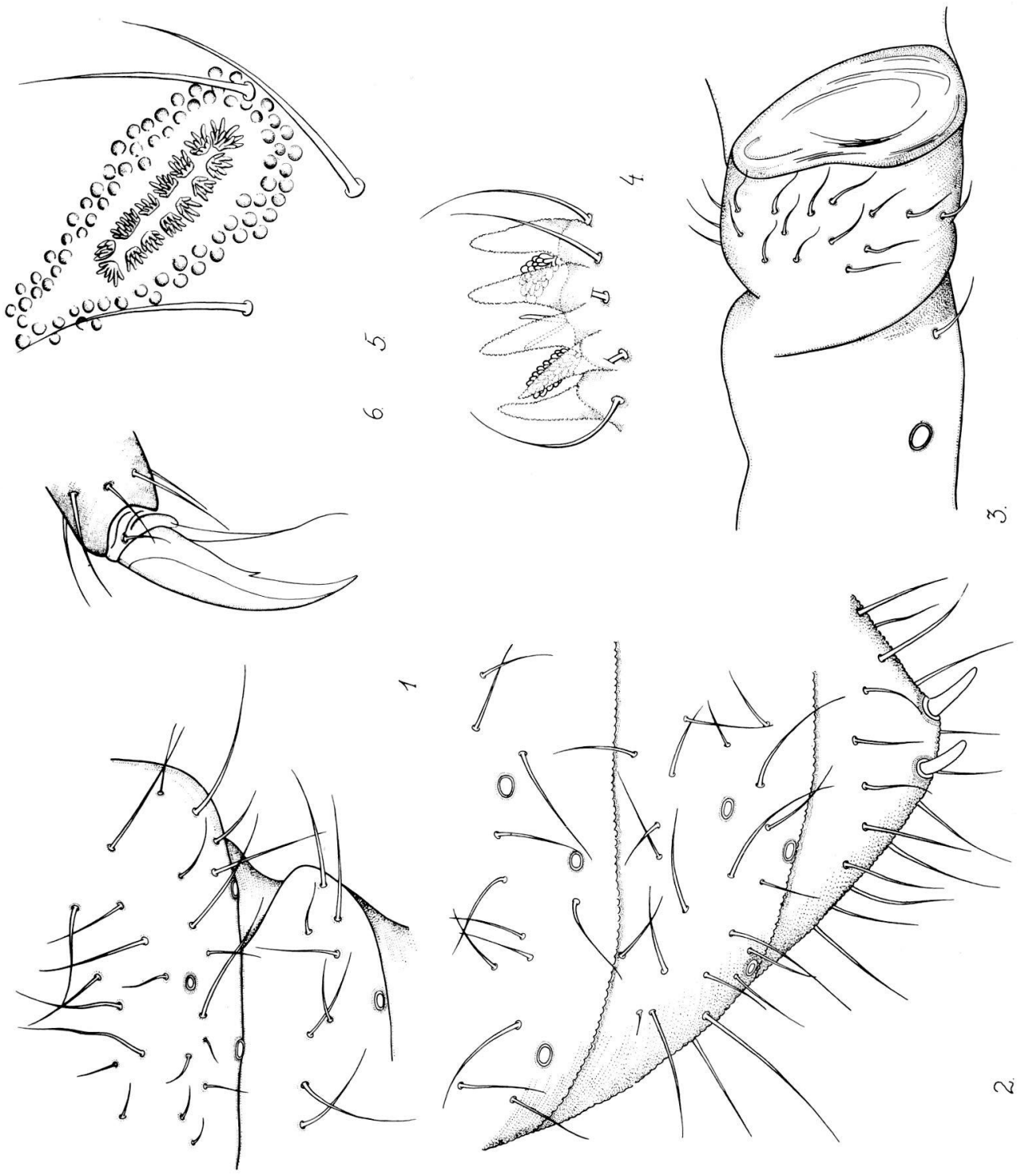


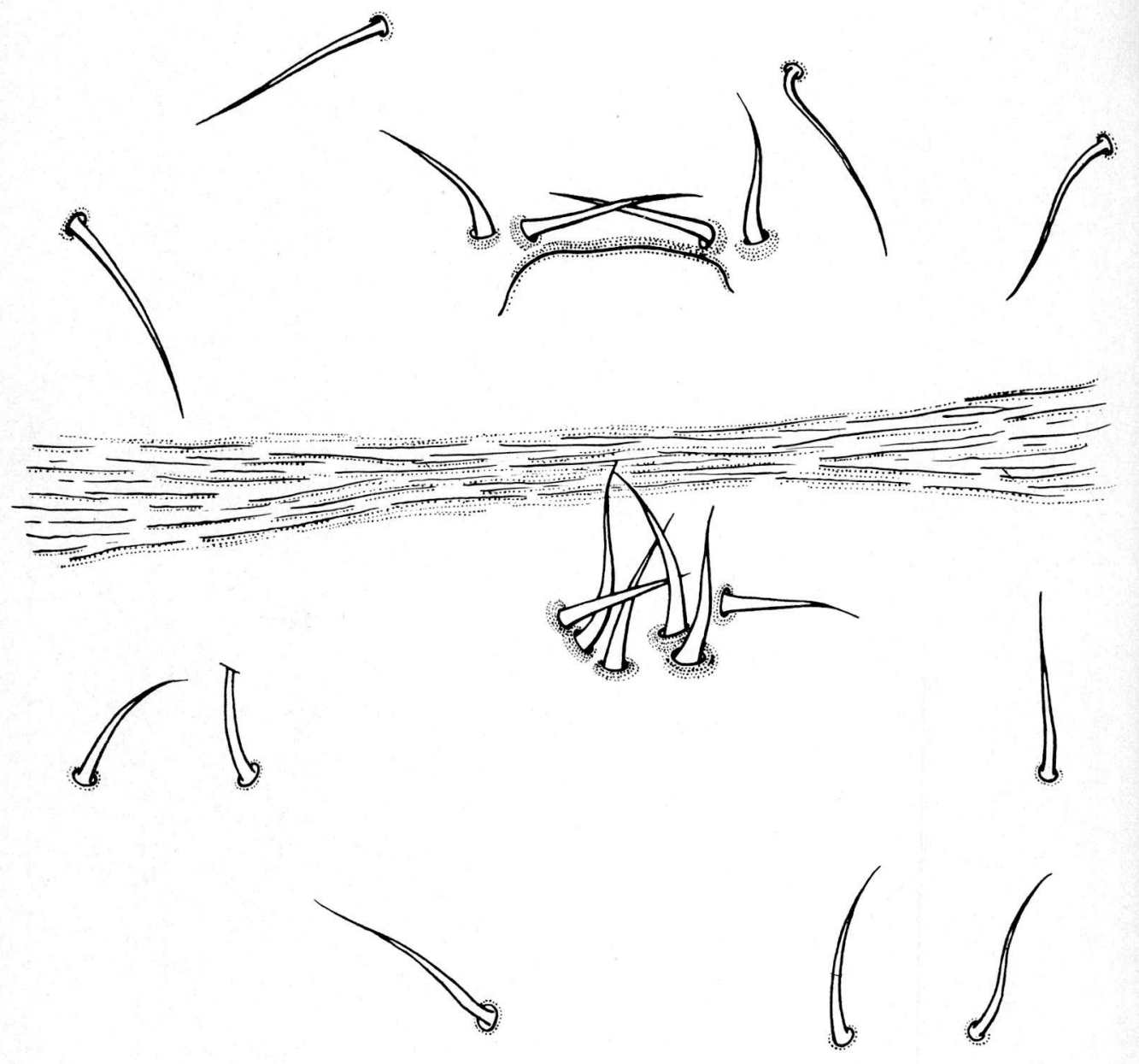

